\title{
Single-Cell Native Mass Spectrometry of Human Erythrocytes
}

\author{
Waka Sakamoto $^{1}$, Nanako Azegami ${ }^{2}$, Tsuyoshi Konuma ${ }^{1,2}$, and Satoko Akashi ${ }^{1,2 *}$ \\ ${ }^{1}$ School of Science, ${ }^{2}$ Graduate School of Medical Life Science, Yokohama City University, 1-7-29 Suehiro- \\ cho, Tsurumi-ku, Yokohama, Kanagawa 230-0045, Japan
}

\section{Supporting information}

\section{Contents}

Experimental Section

Supplementary Table S1, S2, and S3

Supplementary Scheme S1

Supplementary Figures S1, S2, S3, S4, S5, S6, and S7 


\title{
Experimental Section
}

\author{
NanoESI emitters
}

NanoESI emitters were prepared in-house. Borosilicate glass capillaries (GC120-15, Clark Electromedical Instruments, Holliston, MA) were first cut in half, and then pulled with a P-97 capillary puller attached with a box filament FT245B (Sutter Instrument, Novato, CA) (Supplementary Table S3). Pulled capillaries were subsequently processed using Microforge MF-900 (Narishige, Tokyo, Japan) to yield a tip with inner diameter (i.d.) of $\sim 3 \mu \mathrm{m}$, and outer diameter (o.d.) of $\sim 5.5 \mu \mathrm{m}$. The nanoESI emitters were used without metal coating.

Sample preparation for SDS-PAGE and native MS

A Medisafe Finetouch II (Terumo, Japan) was used for sampling human blood from the finger of a consenting healthy volunteer. This was done under the approval of the Ethical Committee of the Graduate School of Medical Life Science, Yokohama City University (Approval Number: 1903). Approximately 50 $\mu \mathrm{L}$ of fresh blood was mixed with $50 \mu \mathrm{L}$ of phosphate buffered saline (PBS) (pH 7.4, Nacalai Tesque, Japan) stored on ice, and centrifuged at $700 \mathrm{~g}$, at $4{ }^{\circ} \mathrm{C}$ for $1 \mathrm{~min}$. Cold PBS $(\sim 50 \mu \mathrm{L})$ was added to the precipitated cells, and the solution was stirred mildly and centrifuged. The precipitated cells were then recovered. This cell washing process was repeated three to four times.

For SDS-PAGE, an aliquot of whole blood or the washed blood cells was mixed with MilliQ water to obtain different $\mathrm{Hb}$ concentrations. After tapping the sample tube to facilitate hemolysis, samples were mixed with SDS-PAGE sample buffer ( $\mathrm{pH}$ 6.8, Nacalai Tesque) at 1:1 (v/v) ratio, and applied to 16\% polyacrylamide gel for electrophoresis.

To extract the blood cell contents for nanoESI-MS, an aliquot of the precipitated blood cells was 
mixed with $500 \mathrm{mM}$ ammonium acetate (stored on ice) at 1:99 (v/v) ratio in a tube. To facilitate hemolysis, the sample tube was tapped with a finger. For native MS, the sample solutions were further diluted with 500 $\mathrm{mM}$ ammonium acetate by 4,40 , and 400 -fold, resulting in $4 \times 10^{2}, 4 \times 10^{3}$, and $4 \times 10^{4}$-fold dilution of the cells. For nanoESI-MS of the $\mathrm{Hb}$ solution, a $3 \mu \mathrm{L}$ aliquot of the sample solution was transferred to the nanoESI emitter fabricated as described previously. The concentrations of $\mathrm{Hb}$ in the sample solutions prepared by 100 - and 400 -fold dilution were confirmed by measuring the absorbance at $538 \mathrm{~nm}(\varepsilon=51,712$ $\mathrm{L} / \mathrm{mol} \cdot \mathrm{cm}$ ), which is specific for oxyhemoglobin derived from a porphyrin structure.

The procedure described above is summarized in Supplementary Scheme S1a.

\section{Aspiration of blood cells for nanoESI-MS}

The solution for cell suspension was first examined. We tested if hemolysis occurred in aqueous solutions of $154 \mathrm{mM}$ sodium acetate, $154 \mathrm{mM}$ potassium acetate, $154 \mathrm{mM}$ ammonium chloride, $85 \mathrm{mM}$ sodium citrate, $85 \mathrm{mM}$ potassium citrate, and $278 \mathrm{mM}$ glucose, by monitoring the absorbance at $538 \mathrm{~nm}$ (Supplementary Table S1). Next, the effects of these compounds on the nanoESI-MS were investigated (Supplementary Figure S1). Subsequently, examination for lowest possible concentrations of sodium acetate, potassium acetate, and glucose was performed by monitoring the absorbance at $538 \mathrm{~nm}$ (Supplementary Table S2). As per the results, $90 \mathrm{mM}$ potassium acetate was used for erythrocyte suspension.

To directly sample the red blood cells for nanoESI-MS, $1 \mu \mathrm{L}$ of cells were suspended in 300-600 $\mu \mathrm{L}$ of an aqueous solution of $90 \mathrm{mM}$ potassium acetate, and a drop of the suspended solution was dispensed on a glass slide. The cells were then aspirated into a nanoESI emitter (details are indicated below) using a micromanipulator (MM-94 with MMO-4, Narishige, Tokyo, Japan) and a microinjector (IM-11-2, Narishige). The aspiration process was monitored and recorded using an inverted microscope (DMIL LED, 
Leica Microsystems, Wetzlar, Germany). The number of blood cells aspirated into the nanoESI emitters was counted manually on-site, by slowly running the movie later, or by comparing the still images of the glass slide before and after cell aspiration. After the cells were sampled, a small aliquot $(<100 \mathrm{~nL})$ of 500 or 100 $\mathrm{mM}$ ammonium acetate was aspirated into the emitter. Subsequently, $3 \mu \mathrm{L}$ of 500 or $100 \mathrm{mM}$ ammonium acetate was infused into the emitter from the rear end, and centrifuged for a few seconds using a microcentrifuge. The sample was then subjected to nanoESI-MS.

The procedure described above is summarized in Supplementary Scheme S1b.

\section{NanoESI-MS}

Mass spectra of $\mathrm{Hb}$ were acquired using a Synapt G2 HDMS mass spectrometer (Waters, Milford, MA) equipped with a nanoESI source. ${ }^{11}$ The nanoESI emitter was placed in the nanospray ion source. The sample solution was pressurized using nitrogen gas from the rear end of the emitter to generate a stable rate of sample flow toward the tip. To apply the ionization voltage, a platinum wire $(0.127 \mathrm{~mm}$, Sigma-Aldrich, St. Louis, MO) was inserted into the nanoESI emitter, as shown in Supplementary Figure S2. The following parameters were applied to obtain the mass spectra: $0.65-0.8 \mathrm{kV}$ capillary voltage for ionization, $20-30 \mathrm{~V}$ sampling cone voltage, $20 \mathrm{~V}$ trap collision energy, and 3.0 L/min Ar gas flow in the trap region. The backing pressure of the ion source was set to $\sim 4$ mbar. The $m / z$ values were calibrated with $(\mathrm{CsI})_{\mathrm{n}} \mathrm{Cs}^{+}$ion clusters. Mass spectra from $m / z 1000$ to 6000 were acquired and processed with MassLynx 4.2. Each mass spectrum was obtained by accumulation for 1 or $2 \mathrm{~min}$. 


\section{Supplementary Tables S1, S2, and S3}

Table S1. Concentration of lysed $\mathrm{Hb}$ in aqueous solutions containing various salts/glucose ${ }^{\mathrm{a}}$

\begin{tabular}{|c|c|c|c|}
\hline & \multicolumn{3}{|c|}{ Concentration of lysed $\mathrm{Hb}(\mu \mathrm{M})$ estimated with absorbance at $538 \mathrm{~nm}$} \\
\hline & $+30 \mathrm{~min}$ & $+60 \mathrm{~min}$ & $+90 \min$ \\
\hline $154 \mathrm{mM}$ Sodium acetate & 1.9 & 2.9 & 3.4 \\
\hline $154 \mathrm{mM}$ Potassium acetate & 3.2 & 4.6 & 5.0 \\
\hline 154 mM Ammonium chloride & 7.0 & 136 & N.T. ${ }^{b}$ \\
\hline $85 \mathrm{mM}$ Sodium citrate & 1.2 & 2.5 & 2.8 \\
\hline $85 \mathrm{mM}$ Potassium citrate & 0.8 & 2.7 & 2.8 \\
\hline $278 \mathrm{mM}$ glucose & N.D. ${ }^{c}$ & 2.9 & 3.9 \\
\hline
\end{tabular}


Table S2. Concentration of lysed $\mathrm{Hb}$ in aqueous solutions containing a low concentration of sodium acetate, potassium acetate, or glucose ${ }^{a}$

\begin{tabular}{lccc}
\hline & $\begin{array}{c}\text { Concentration } \\
(\mathrm{mM})\end{array}$ & Concentration of lysed $\mathrm{Hb}(\mu \mathrm{M})$ estimated with absorbance at 538 nm \\
\cline { 3 - 4 } Sodium acetate & 90 & $+30 \mathrm{~min}$ & $+60 \mathrm{~min}$ \\
& 85 & 1.8 & 2.4 \\
& 80 & 2.8 & 3.6 \\
& 78 & 7.9 & 14.1 \\
\hline Potassium acetate & 90 & 22.2 & N.T. ${ }^{\mathrm{b}}$ \\
& 85 & 1.4 & 1.3 \\
& 80 & 4.0 & 4.8 \\
& 78 & 9.6 & 11.9 \\
\hline Glucose & 163 & 20.0 & N.T. \\
& 158 & 2.7 & 3.0 \\
& 154 & 2.8 & 4.3 \\
& 150 & 4.9 & 6.2 \\
\hline
\end{tabular}

a $2 \mu \mathrm{L}$ of blood cells was mixed with $38 \mu \mathrm{L}$ of solution. If complete hemolysis occurs, the concentration of $\mathrm{Hb}$ is $\sim 250 \mu \mathrm{M}$.

${ }^{\mathrm{b}}$ Not tested.

Table S3. Program for preparation of nanoESI emitters with P-97 Puller

\begin{tabular}{cccccc}
\hline & Heat & Pull & Velocity & Time & Pressure \\
\hline Line 1 & $742^{\mathrm{a}}$ & 20 & 8 & 250 & 500 \\
Line 2 & 742 & 20 & 8 & 250 & 500 \\
Line 3 & 742 & 50 & 15 & 250 & 500 \\
\hline
\end{tabular}

${ }^{\text {a }}$ Corresponding to the (ramp-2) value. 


\section{Supplementary Scheme S1}

(a) Blood $\sim 50 \mu \mathrm{L}$ : Sampled using Medisafe Finetouch II

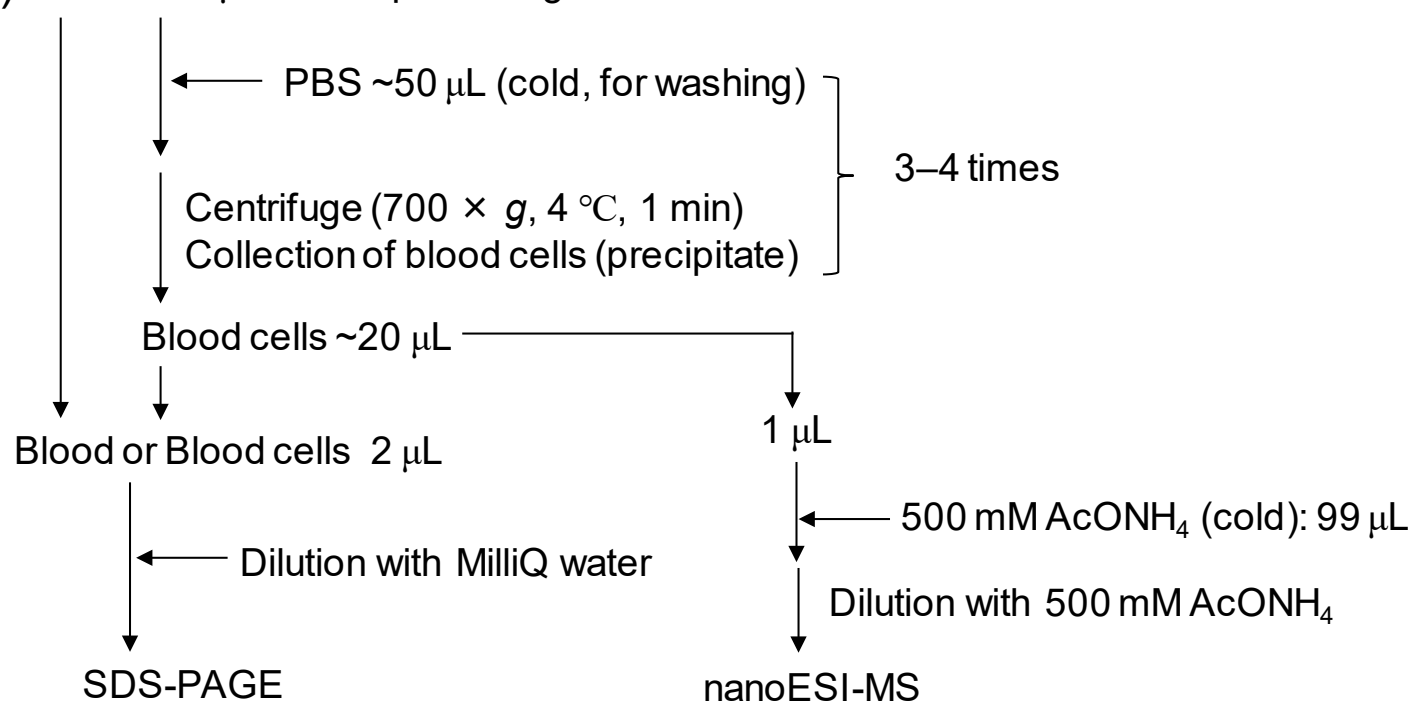

(b) Blood $\sim 50 \mu \mathrm{L}$ : Sampled using Medisafe Finetouch II

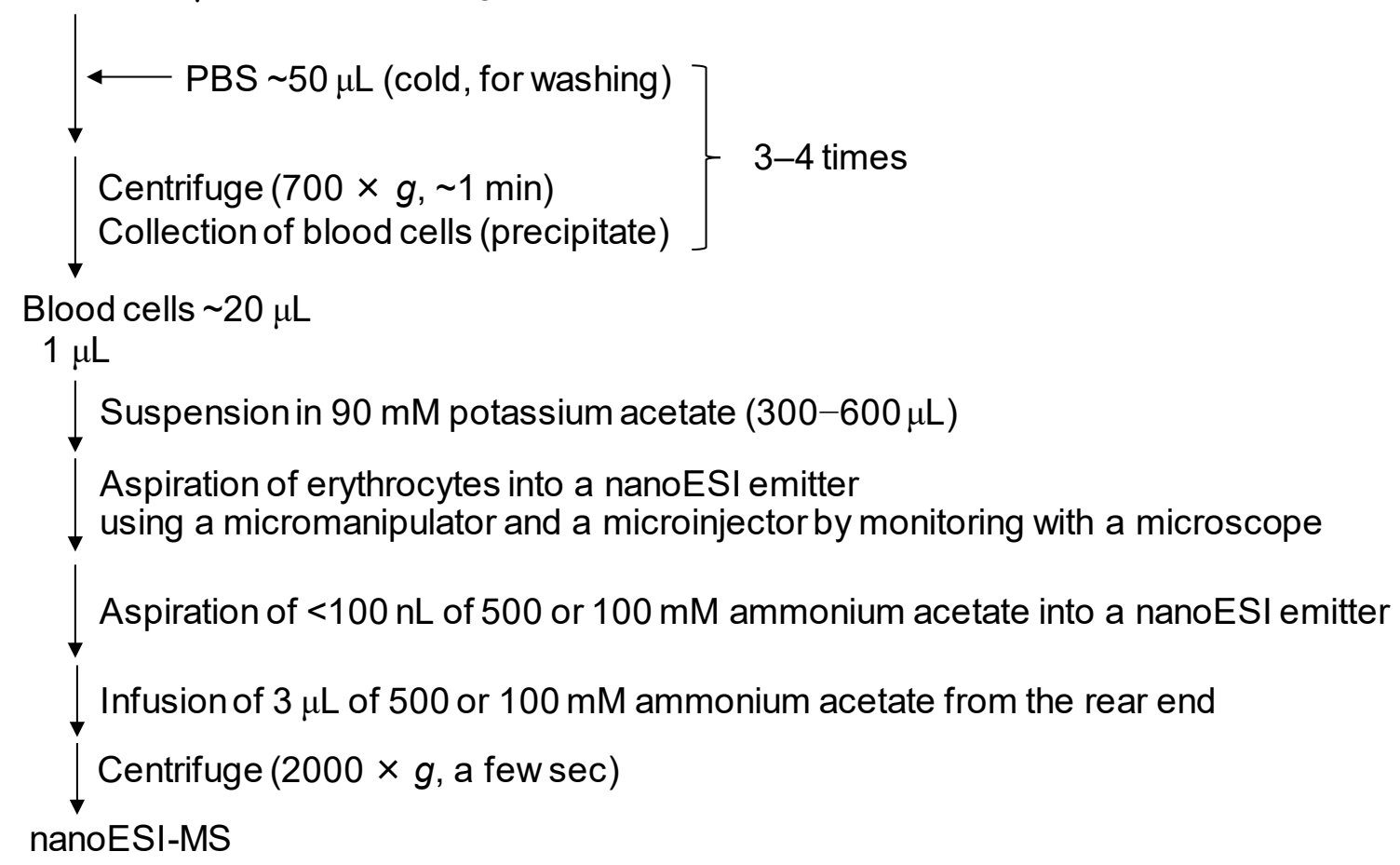

Scheme S1. Preparation procedure for (a) SDS-PAGE and nanoESI-MS samples by dilution of blood and blood cells in a microtube and (b) nanoESI-MS samples by aspiration of red blood cells into a nanoESI emitter. 


\section{Supplementary Figures S1, S2, S3, S4, S5, S6, and S7}

a) Sodium acetate

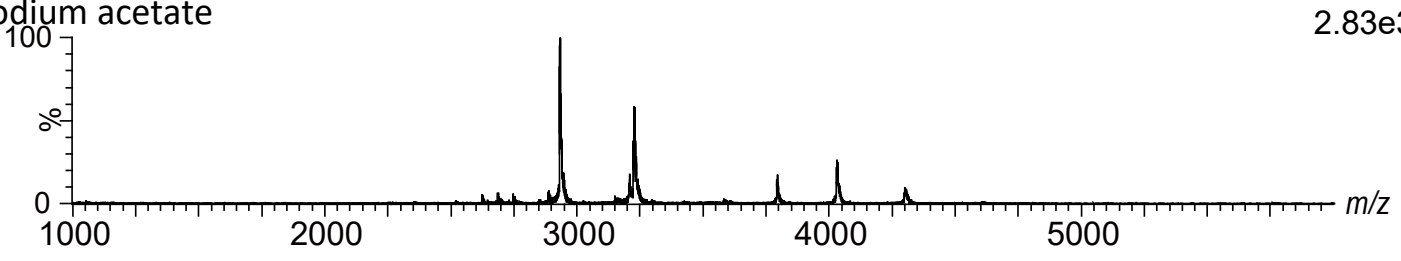

b) Potassium acetate

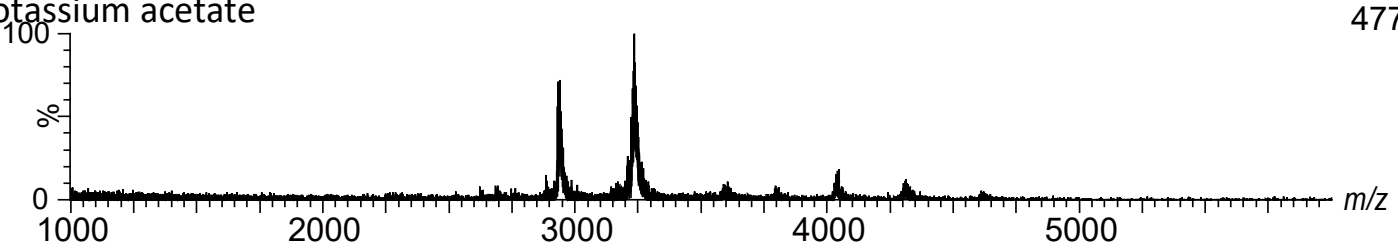

c) Sodium citrate

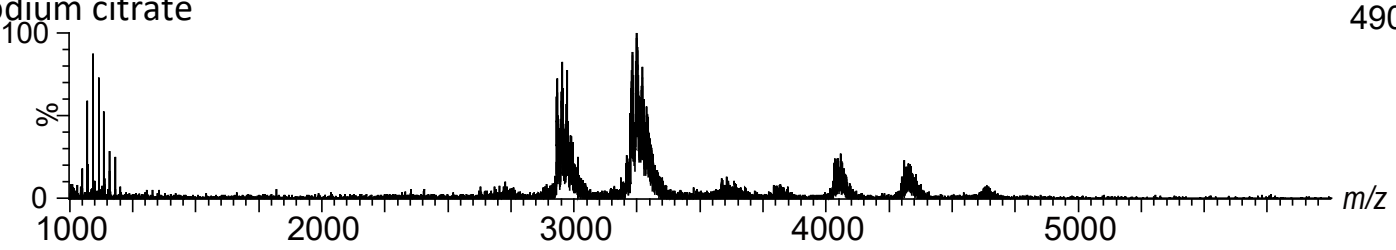

d) Potassium citrate

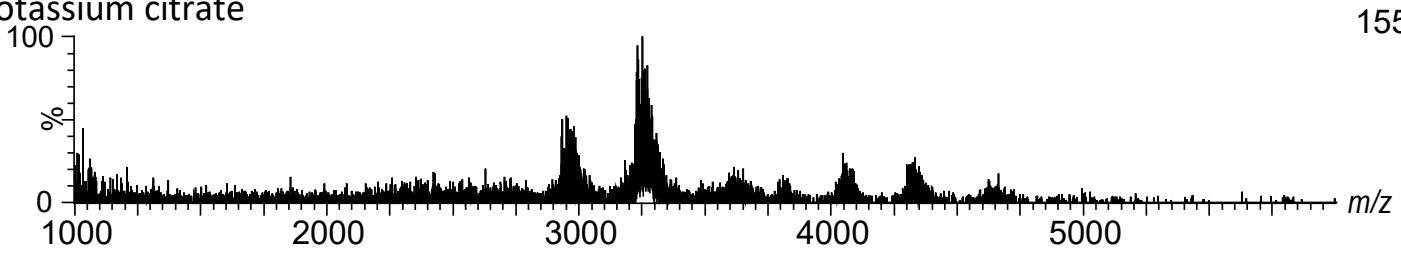

e) Glucose

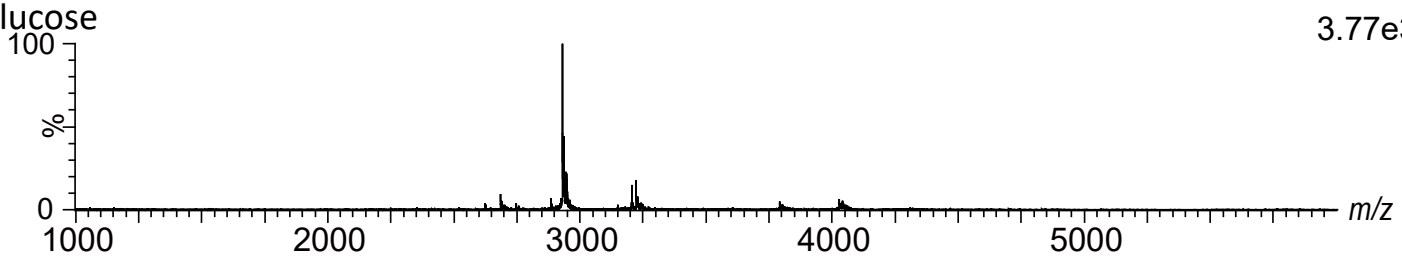

Figure S1. NanoESI mass spectra of blood cell samples prepared by 20 -fold dilution with various aqueous solutions: (a) $154 \mathrm{mM}$ sodium acetate, (b) $154 \mathrm{mM}$ potassium acetate, (c) $85 \mathrm{mM}$ sodium citrate, (d) $85 \mathrm{mM}$ potassium citrate, and (e) $278 \mathrm{mM}$ glucose. The 20 -fold diluted solutions of blood cells were mixed with $500 \mathrm{mM}$ ammonium acetate at a 1:99 ratio, and subjected to nanoESI-MS. 


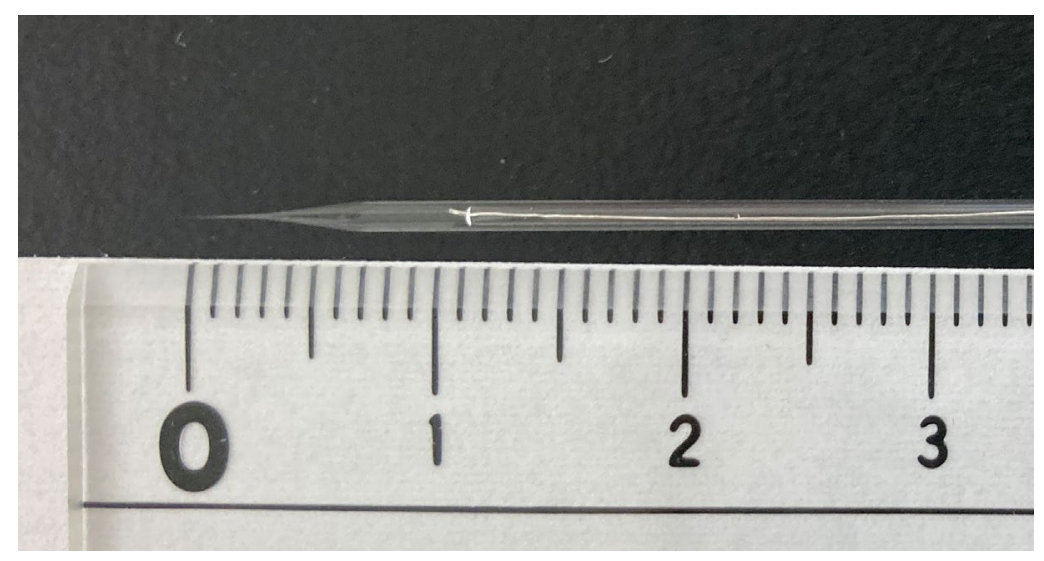

Figure S2. Image of a nanoESI emitter containing $3 \mu \mathrm{L}$ of the solution with a Pt wire for nanoESI voltage application. Each small division indicates $1 \mathrm{~mm}$.

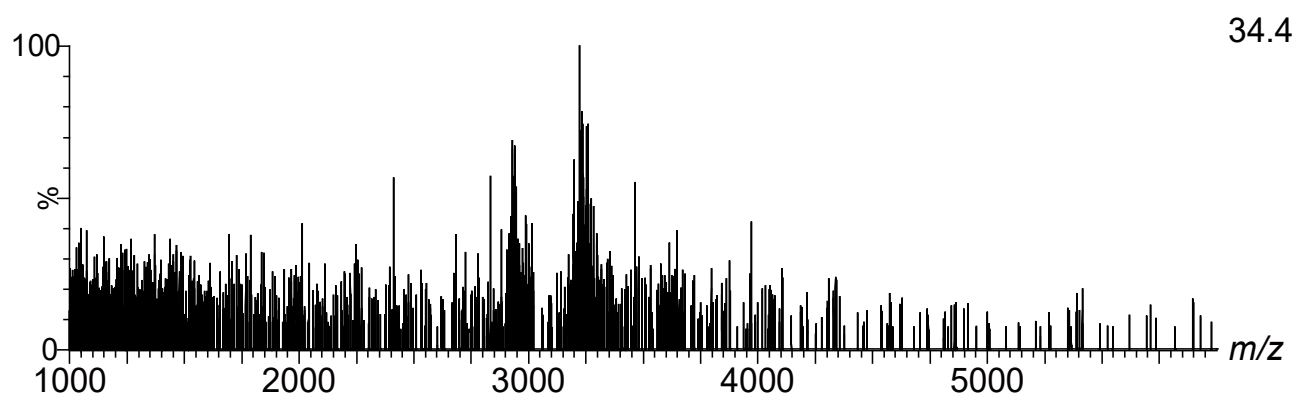

Figure S3. NanoESI mass spectra of the samples prepared with five aspirated erythrocytes. 
a) $10 \mathrm{mM}$ ammonium acetate

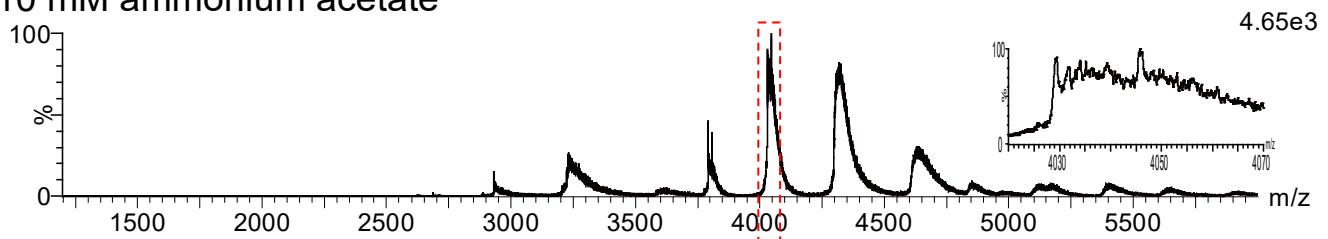

b) $100 \mathrm{mM}$ ammonium acetate

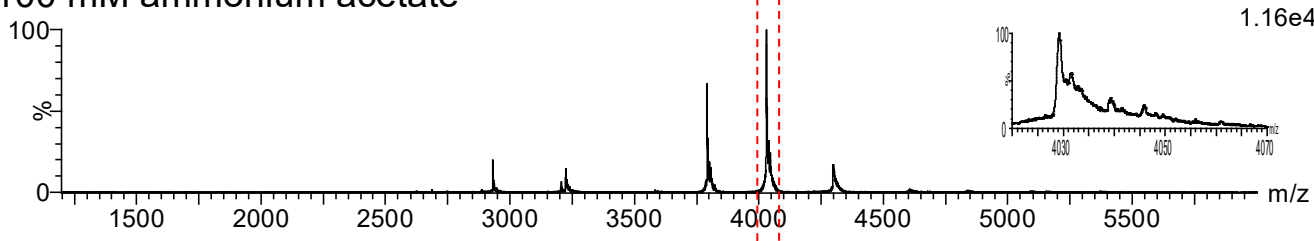

c) $500 \mathrm{mM}$ ammonium acetate

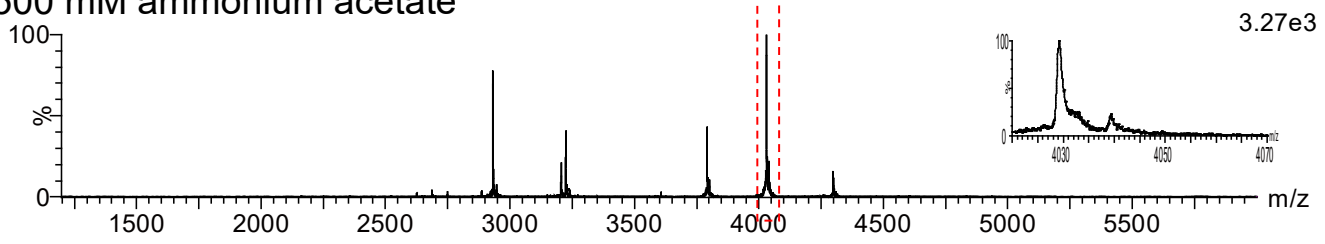

Figure S4. NanoESI mass spectra of blood cell lysates prepared with (a) $10 \mathrm{mM}$, (b) $100 \mathrm{mM}$, and (c) $500 \mathrm{mM}$ ammonium acetate. Insets are magnified mass spectra from $m / z 4020$ to 4070 .

a) $500 \mathrm{mM}$ ammonium acetate

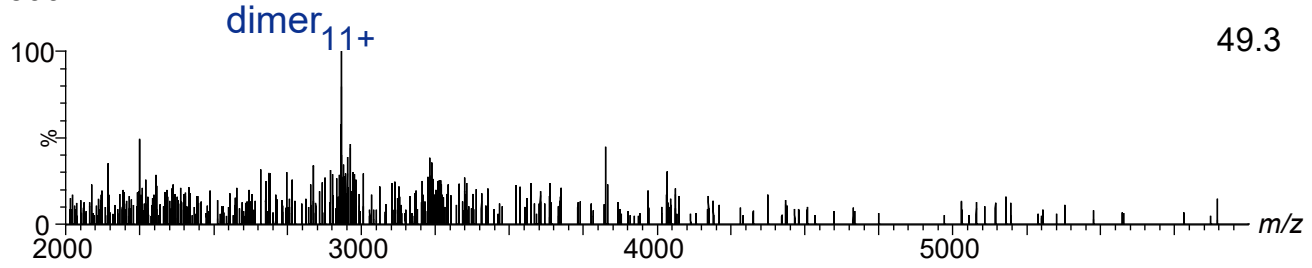

b) $200 \mathrm{mM}$ ammonium acetate

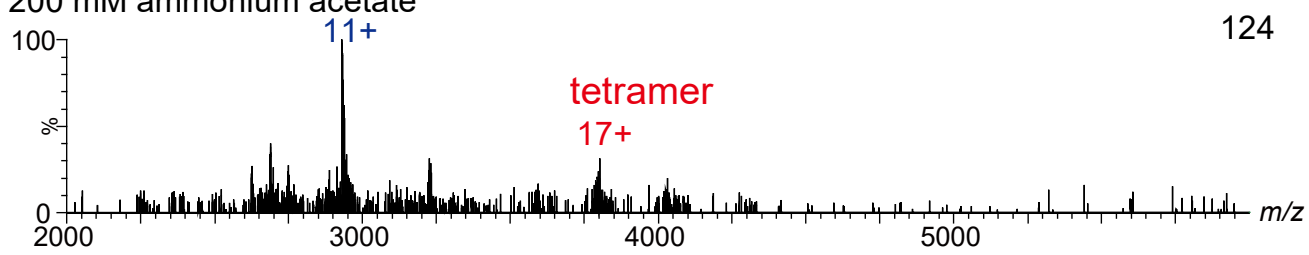

c) $100 \mathrm{mM}$ ammonium acetate

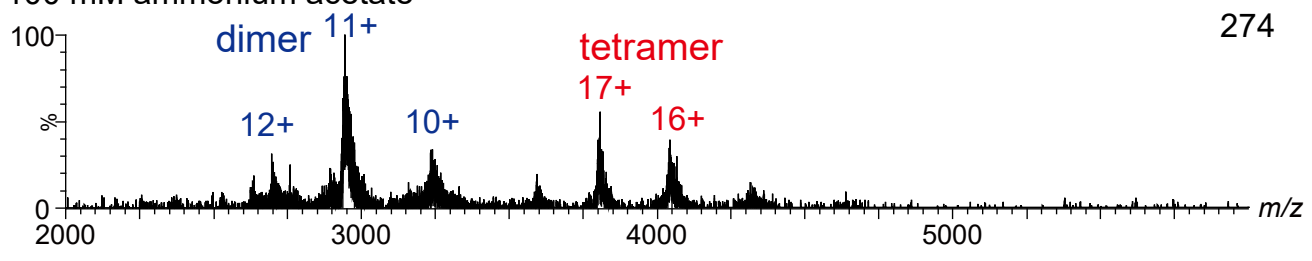

Figure S5. NanoESI mass spectra of five aspirated erythrocytes prepared with (a) $500 \mathrm{mM}$, (b) $200 \mathrm{mM}$, and (c) $100 \mathrm{mM}$ ammonium acetate. 


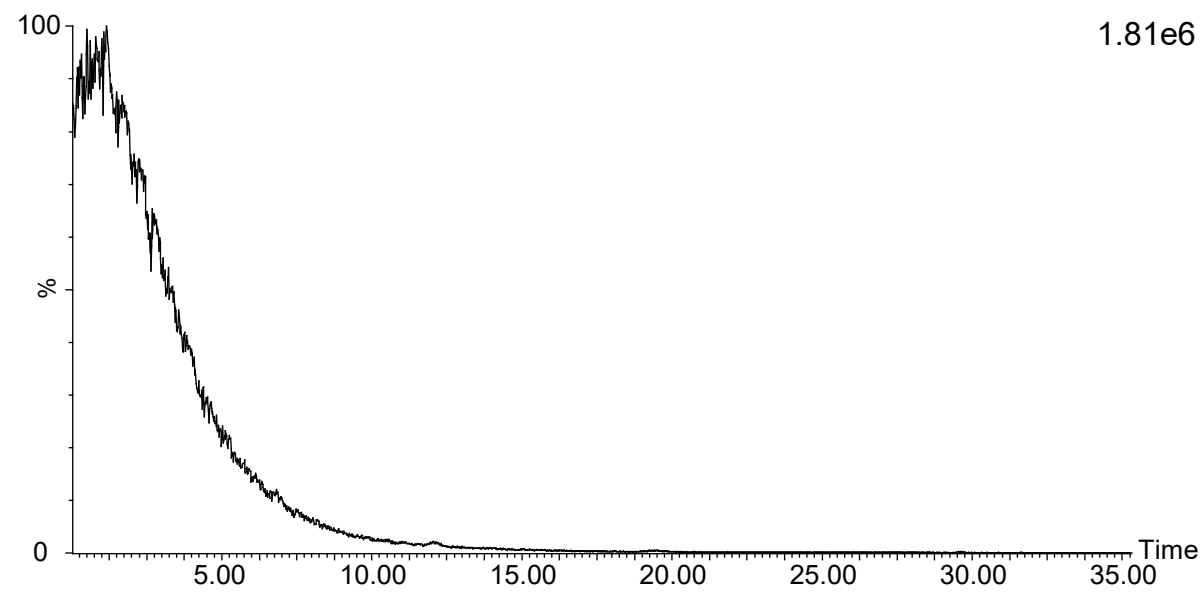

Figure S6. Total ion chromatogram of nanoESI mass spectrometry for a sample of $\sim 300$ red blood cells.

a)

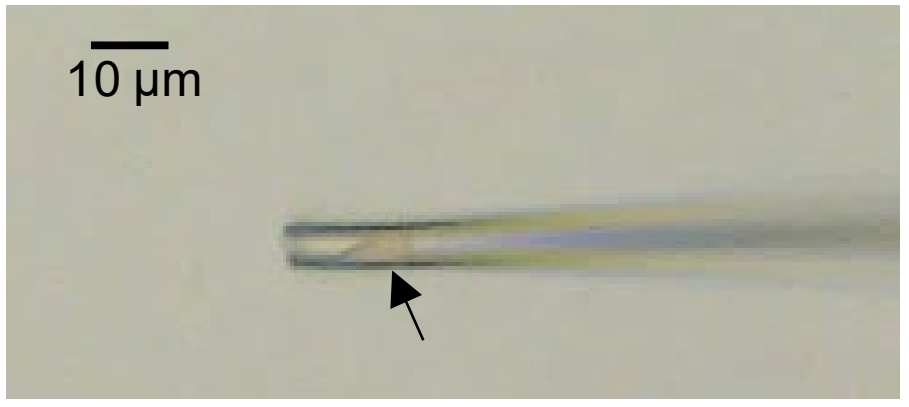

b)

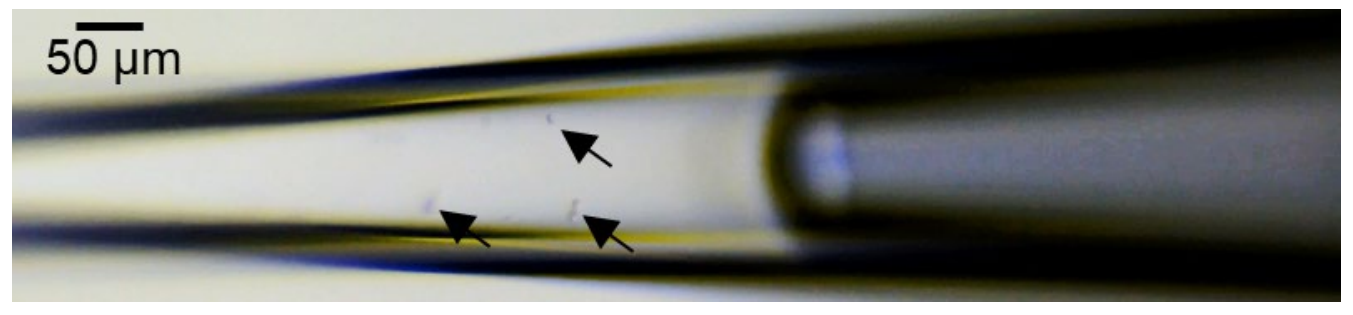

Figure S7 Images of nanoESI emitters after aspiration of erythrocytes. (a) A snapshot of the emitter immediately after the sampling of 221 blood cells from $90 \mathrm{mM}$ potassium acetate. The arrowhead indicates the location of erythrocytes aspirated into the emitter. The tip of the emitter has been focused on. (b) A snapshot of the emitter at 2-min aspiration of the ammonium acetate solution $(<100 \mathrm{~nL})$ following erythrocyte sampling. The upper surface of the solution in the emitter has been focused on. Arrowheads indicate the locations of erythrocytes. 